\title{
Some results on uniqueness of meromorphic functions sharing a polynomial
}

\author{
Pulak Sahoo ${ }^{1}$ and Biswajit Saha ${ }^{2}$ \\ ${ }^{1}$ Department of Mathematics, University of Kalyani, West Bengal-741235, India. \\ ${ }^{2}$ Department of Mathematics, Govt. College of Engineering and Textile Technology, Serampore, West Bengal-712201, \\ India. \\ E-mail: sahoopulak@yahoo. $\mathrm{com}^{1}$, sahoopulak1@gmail.com ${ }^{1}$, sahaanjan11@gmail.com ${ }^{2}$
}

\begin{abstract}
In this paper, with the aid of weighted value sharing we study the uniqueness problems of meromorphic functions when certain nonlinear differential polynomials generated by them share a nonconstant polynomial with weight two. The result of the paper not only improves the results due to the present first author [Bull. Math. Anal. Appl., 2(2010), 106-118] and of Zhang and $\mathrm{Xu}$ [Comput. Math. Appl., 61(2011), 722-730], at the same time finds a possible answer of an open question posed by Zhang and Xu.
\end{abstract}

2010 Mathematics Subject Classification. 30D35.

Keywords. Uniqueness, meromorphic function, weighted sharing.

\section{Introduction, definitions and results}

In this paper, by meromorphic functions we will always mean meromorphic functions in the complex plane. We assume that the reader is familiar with the classical value distribution theory of meromorphic functions as described in, say, the standard monograph $[6,16]$. For a nonconstant meromorphic function $f$, we denote by $T(r, f)$ the Nevanlinna characteristic of $f$ and by $S(r, f)$ any quantity satisfying $S(r, f)=o\{T(r, f)\}$ as $r \rightarrow \infty$ outside of a possible exceptional set E of finite linear measure. The meromorphic function $a(z)$ is called a small function of $f$ if $T(r, a)=S(r, f)$.

Two nonconstant meromorphic functions $f$ and $g$ share a small function $a$ CM (counting multiplicities) provided that $f-a$ and $g-a$ have the same set of zeros with the same multiplicities; $f$ and $g$ share $a$ IM (ignoring multiplicities) if we do not consider the multiplicities. A finite value $z_{0}$ is called a fixed point of $f(z)$ if $f\left(z_{0}\right)=z_{0}$.

The following result is very well known in the value distribution theory (see $[3,4])$.

Theorem A. Let $f$ be a transcendental meromorphic function, and let $n(\geq 1)$ be an integer. Then $f^{n} f^{\prime}=1$ has infinitely many solutions.

Corresponding to Theorem A the following result was proved by Yang and Hua [14] in 1997.

Theorem B. Let $f$ and $g$ be two nonconstant meromorphic functions, and let $n(\geq 11)$ be an integer. If $f^{n} f^{\prime}$ and $g^{n} g^{\prime}$ share the value $1 \mathrm{CM}$, then either $f(z)=c_{1} e^{c z}, g(z)=c_{2} e^{-c z}$, where $c_{1}, c_{2}$ and $c$ are three constants satisfying $\left(c_{1} c_{2}\right)^{n+1} c^{2}=-1$ or $f=t g$ for a constant $t$ such that $t^{n+1}=1$.

Regarding fixed point the following result was obtained by Fang and Qiu [5] in 2000.

Tbilisi Mathematical Journal 9(2) (2016), pp. 59-70.

Tbilisi Centre for Mathematical Sciences.

Received by the editors: 23 March 2016.

Accepted for publication: 05 August 2016 
Theorem C. Let $f$ and $g$ be two nonconstant meromorphic functions, and let $n(\geq 11)$ be an integer. If $f^{n} f^{\prime}$ and $g^{n} g^{\prime}$ share $z \mathrm{CM}$, then either $f(z)=c_{1} e^{c z^{2}}, g(z)=c_{2} e^{-c z^{2}}$, where $c_{1}, c_{2}$ and $c$ are three nonzero complex constants satisfying $4\left(c_{1} c_{2}\right)^{n+1} c^{2}=-1$ or $f=t g$ for a complex number $t$ satisfying $t^{n+1}=1$.

A new trend in this direction is to consider the uniqueness of a meromorphic functions concerning the value sharing of the $k$-th derivative of a linear expression of a meromorphic function. For the last decade a handful number of astonishing results have been obtained regarding the value sharing of nonlinear differential polynomials which are mainly the $k$-th derivative of some linear expressions of $f$ and $g$ (see [8], [9], [10] and [11], for example). In $2010 \mathrm{Xu}, \mathrm{Lu}$ and $\mathrm{Yi}$ [12] proved the following results for meromorphic functions where an additional condition namely the sharing of poles by the meromorphic functions are taken into consideration.

Theorem D. Let $f$ and $g$ be two nonconstant meromorphic functions, and let $n, k$ be two positive integers with $n>3 k+10$. If $\left(f^{n}\right)^{(k)}$ and $\left(g^{n}\right)^{(k)}$ share $z \mathrm{CM}, f$ and $g$ share $\infty$ IM, then either $f(z)=c_{1} e^{c z^{2}}, g(z)=c_{2} e^{-c z^{2}}$, where $c_{1}, c_{2}$ and $c$ are three constants satisfying $4 n^{2}\left(c_{1} c_{2}\right)^{n} c^{2}=-1$ or $f=t g$ for a constant $t$ such that $t^{n}=1$.

Theorem E. Let $f$ and $g$ be two nonconstant meromorphic functions satisfying $\Theta(\infty, f)>\frac{2}{n}$, and let $n, k$ be two positive integers with $n \geq 3 k+12$. If $\left(f^{n}(f-1)\right)^{(k)}$ and $\left(g^{n}(g-1)\right)^{(k)}$ share $z$ CM, $f$ and $g$ share $\infty$ IM, then $f=g$.

Observing the above results the following question is inevitable.

Question 1. Is it possible in any way to relax the nature of sharing the fixed point and in the same time reduce the lower bound of $n$ in Theorems $\mathrm{D}$ and $\mathrm{E}$ ?

To proceed further we need the following notion of weighted sharing of values which measures how close a shared value is to being shared CM or to being shared IM.

Definition 1. [7] Let $k$ be a nonnegative integer or infinity. For $a \in \mathbb{C} \cup\{\infty\}$ we denote by $E_{k}(a ; f)$ the set of all $a$-points of $f$ where an $a$-point of multiplicity $\mathrm{m}$ is counted $\mathrm{m}$ times if $m \leq k$ and $\mathrm{k}+1$ times if $m>k$. If $E_{k}(a ; f)=E_{k}(a ; g)$, we say that $f, g$ share the value $a$ with weight $\mathrm{k}$.

The definition implies that if $f, g$ share a value $a$ with weight $k$, then $z_{0}$ is an $a$-point of $f$ with multiplicity $m(\leq k)$ if and only if it is an $a$-point of $g$ with multiplicity $m(\leq k)$ and $z_{0}$ is an a-point of $f$ with multiplicity $m(>k)$ if and only if it is an a-point of $g$ with multiplicity $n(>k)$, where $\mathrm{m}$ is not necessarily equal to $\mathrm{n}$.

We write $f, g$ share $(a, k)$ to mean that $f, g$ share the value $a$ with weight $\mathrm{k}$. Clearly if $f, g$ share $(a, k)$ then $f, g$ share $(a, p)$ for any integer $p, 0 \leq p<k$. Also we note that $f, g$ share a value $a$ IM or CM if and only if $f, g$ share $(a, 0)$ or $(a, \infty)$ respectively.

Keeping in mind the notion of weighted sharing of values, the present first author [9] proved the following result in 2010 which deals with Question 1.

Theorem F. Let $f$ and $g$ be two transcendental meromorphic functions, and let $n, k$ and $m$ be three positive integers satisfying $n>3 k+m+7$. Suppose that $P(w)=a_{m} w^{m}+\ldots+a_{1} w+a_{0}$, where $a_{0}(\neq 0), a_{1}, \ldots, a_{m}(\neq 0)$ are complex constants. If $\left[f^{n} P(f)\right]^{(k)}$ and $\left[g^{n} P(g)\right]^{(k)}$ share $(z, 2) ; f$ and $g$ share $(\infty, 0)$ then either $f=t g$ for a constant $t$ such that $t^{d}=1$, where $d=$ 
$\operatorname{gcd}(n+m, \ldots, n+m-i, \ldots, n), a_{m-i} \neq 0$ for some $i=0,1,2, \ldots, m$ or $f$ and $g$ satisfy the algebraic equation $R(f, g)=0$, where

$$
R\left(w_{1}, w_{2}\right)=w_{1}^{n}\left(a_{m} w_{1}^{m}+\ldots+a_{1} w_{1}+a_{0}\right)-w_{2}^{n}\left(a_{m} w_{2}^{m}+\ldots+a_{1} w_{2}+a_{0}\right) .
$$

It is now quite natural to ask the following question.

Question 2. What can be said if sharing fixed point in Theorem $\mathrm{F}$ is replaced by sharing a nonzero polynomial ?

In 2011 Zhang and Xu [18] obtained the following result in which they replace fixed point sharing by sharing a nonzero polynomial with degree $\leq 5$.

Theorem G. Let $f$ and $g$ be two transcendental meromorphic functions, let $p(z)$ be a nonzero polynomial with $\operatorname{deg}(p)=l \leq 5, n, k$ and $m$ be three positive integers with $n>3 k+m+7$. Let $P(w)=a_{m} w^{m}+\ldots+a_{1} w+a_{0}$ be a nonzero polynomial. If $\left[f^{n} P(f)\right]^{(k)}$ and $\left[g^{n} P(g)\right]^{(k)}$ share $p(z)$ CM, $f$ and $g$ share $\infty$ IM, then one of the following three cases hold:

(i) $f=t g$ for a constant $t$ such that $t^{d}=1$, where $d=\operatorname{gcd}(n+m, \ldots, n+m-i, \ldots, n), a_{m-i} \neq 0$ for some $i=0,1,2, \ldots, m$;

(ii) $f$ and $g$ satisfy the algebraic equation $R(f, g)=0$, where $R(f, g)$ is given by (1.1);

(iii) $P(w)$ is reduced to a nonzero monomial, namely, $P(w)=a_{i} w^{i} \not \equiv 0$ for some $i \in\{0,1, \ldots, m\}$; if $p(z)$ is not a constant, then $f(z)=c_{1} e^{c Q(z)}, g(z)=c_{2} e^{-c Q(z)}$, where $Q(z)=\int_{0}^{z} p(z) d z, c_{1}, c_{2}$ and $c$ are three constants satisfying $a_{i}^{2}\left(c_{1} c_{2}\right)^{n+i}[(n+i) c]^{2}=-1$, if $p(z)$ is a nonzero constant $b$, then $f(z)=c_{3} e^{c z}, g(z)=c_{4} e^{-c z}$, where $c_{3}, c_{4}$ and $c$ are three constants such that $(-1)^{k} a_{i}^{2}\left(c_{3} c_{4}\right)^{n+i}[(n+$ i)c $]^{2 k}=b^{2}$.

In the same paper the authors posed the following question, as far as we are aware, this remains open.

Question 3. Is it possible in any way to remove the condition $\operatorname{deg}(p)=l \leq 5$ in Theorem G ?

In the paper our main concern is to find out the possible answer of the above question. The result of the paper improves Theorem $\mathrm{F}$ by reducing the lower bound of $n$ and also improves Theorem G by relaxing the nature of sharing the polynomial as well as by reducing the lower bound of $n$. The following is the main result of the paper.

Theorem 1. Let $f$ and $g$ be two transcendental meromorphic functions, $p(z)$ be a nonconstant polynomial of degree $l$, and let $n(\geq 1), k(\geq 1)$ and $m(\geq 0)$ be three integers with $n>\max \{3 k+$ $m+6, k+2 l\}$. Suppose that either $k, l$ are co-prime or $k>l$ when $l \geq 2$. Let $P(w)$ be defined as in Theorem G. If $\left[f^{n} P(f)\right]^{(k)}-p$ and $\left[g^{n} P(g)\right]^{(k)}-p$ share $(0,2) ; f$ and $g$ share $(\infty, 0)$, then the following conclusions hold:

(i) If $P(w)=a_{m} w^{m}+\ldots+a_{1} w+a_{0}$ is not a monomial, then either $f=t g$ for a constant $t$ that satisfies $t^{d}=1$, where $d=(n+m, \ldots, n+m-i, \ldots, n), a_{m-i} \neq 0$ for some $i=0,1,2, \ldots, m$; or $f$ and $g$ satisfy the algebraic equation $R(f, g)=0$, where $R(f, g)$ is given by (1.1).

(ii) When $P(w)=c_{0}$ or $P(w)=a_{m} w^{m}$, then either $f=t g$ for a constant $t$ that satisfies $t^{n+m^{*}}=1$, or $f(z)=b_{1} e^{b Q(z)}, g(z)=b_{2} e^{-b Q(z)}$, where $Q(z)$ is a polynomial without constant such that $Q^{\prime}(z)=$ $p(z), b_{1}, b_{2}$ and $b$ are three constants satisfying $c_{0}^{2}(n b)^{2}\left(b_{1} b_{2}\right)^{n}=-1$ or $a_{m}^{2}((n+m) b)^{2}\left(b_{1} b_{2}\right)^{n+m}=$ -1 , where $m^{*}$ is defined by

$$
m^{*}= \begin{cases}m & \text { if } P(w) \neq c_{0} \\ 0 & \text { if } P(w)=c_{0}\end{cases}
$$


We now explain the following definitions and notations which are used in the paper.

Definition 2. [7] Let $a \in \mathbb{C} \cup\{\infty\}$. We denote by $N(r, a ; f \mid=1)$ the counting function of simple $a$ points of $f$. For a positive integer $p$ we denote by $N(r, a ; f \mid \leq p)$ the counting function of those $a$-points of $f$ (counted with proper multiplicities) whose multiplicities are not greater than $p$. By $\bar{N}(r, a ; f \mid \leq p)$ we denote the corresponding reduced counting function.

Analogously we can define $N(r, a ; f \mid \geq p)$ and $\bar{N}(r, a ; f \mid \geq p)$.

Definition 3. Let $a$ be any value in the extended complex plane, and let $k$ be an arbitrary nonnegative integer. We denote by $N_{k}(r, a ; f)$ the counting function of $a$-points of $f$, where an $a$-point of multiplicity $m$ is counted $m$ times if $m \leq k$ and $k$ times if $m>k$. Then

$$
N_{k}(r, a ; f)=\bar{N}(r, a ; f)+\bar{N}(r, a ; f \mid \geq 2)+\ldots+\bar{N}(r, a ; f \mid \geq k) .
$$

Clearly $N_{1}(r, a ; f)=\bar{N}(r, a ; f)$.

Definition 4. [1] Let $f$ and $g$ be two nonconstant meromorphic functions that share $(a, 2)$ for $a \in \mathbb{C} \cup\{\infty\}$. Let $z_{0}$ be an $a$-point of $f$ with multiplicity $p$ and also an $a$-point of $g$ with multiplicity $q$. We denote by $\bar{N}_{L}(r, a ; f)\left(\bar{N}_{L}(r, a ; g)\right)$ the reduced counting function of those $a$-points of $f$ and $g$, where $p>q \geq 3(q>p \geq 3)$. Also we denote by $\bar{N}_{E}^{(3}(r, a ; f)$ the reduced counting function of those $a$-points of $f$ and $g$, where $p=q \geq 3$.

Definition 5. [7] Let $f$ and $g$ be two nonconstant meromorphic functions sharing the value $a$ IM. We denote by $\bar{N}_{*}(r, a ; f, g)$ the reduced counting function of those $a$-points of $f$ whose multiplicities differ from the multiplicities of the corresponding $a$-points of $g$. Clearly

$$
\bar{N}_{*}(r, a ; f, g)=\bar{N}_{*}(r, a ; g, f)=\bar{N}_{L}(r, a ; f)+\bar{N}_{L}(r, a ; g) .
$$

\section{Lemmas}

Let $F$ and $G$ be two nonconstant meromorphic functions defined in the complex plane $\mathbb{C}$. We denote by $H$ the following function:

$$
H=\left(\frac{F^{\prime \prime}}{F^{\prime}}-\frac{2 F^{\prime}}{F-1}\right)-\left(\frac{G^{\prime \prime}}{G^{\prime}}-\frac{2 G^{\prime}}{G-1}\right) .
$$

Lemma 1. [13] Let $f$ be a nonconstant meromorphic function and let $a_{n}(z)(\not \equiv 0), a_{n-1}(z), \ldots$, $a_{0}(z)$ be meromorphic functions such that $T\left(r, a_{i}(z)\right)=S(r, f)$ for $i=0,1,2, \ldots, n$. Then

$$
T\left(r, a_{n} f^{n}+a_{n-1} f^{n-1}+\ldots+a_{1} f+a_{0}\right)=n T(r, f)+S(r, f) .
$$

Lemma 2. [17] Let $f$ be a nonconstant meromorphic function, and $p, k$ be two positive integers. Then

$$
\begin{gathered}
N_{p}\left(r, 0 ; f^{(k)}\right) \leq T\left(r, f^{(k)}\right)-T(r, f)+N_{p+k}(r, 0 ; f)+S(r, f), \\
N_{p}\left(r, 0 ; f^{(k)}\right) \leq k \bar{N}(r, \infty ; f)+N_{p+k}(r, 0 ; f)+S(r, f) .
\end{gathered}
$$


Lemma 3. [6] Suppose that $f$ is a nonconstant meromorphic function, $k \geq 2$ is an integer. If

$$
N(r, \infty ; f)+N(r, 0 ; f)+N\left(r, 0 ; f^{(k)}\right)=S\left(r, \frac{f^{\prime}}{f}\right),
$$

then $f=e^{a z+b}$, where $a(\neq 0), b$ are constants.

Lemma 4. [2] Let $F, G$ be two nonconstant meromorphic functions sharing $(1,2)$ and $(\infty, k)$ where $0 \leq k<\infty$. If $H \not \equiv 0$, then

(i) $T(r, F) \leq N_{2}(r, 0 ; F)+N_{2}(r, 0 ; G)+\bar{N}(r, \infty ; F)+\bar{N}(r, \infty ; G)+\bar{N}_{*}(r, \infty ; F, G)-m(r, 1 ; G)-$ $\bar{N}_{E}^{(3}(r, 1 ; F)-\bar{N}_{L}(r, 1 ; G)+S(r, F)+S(r, G)$;

(ii) $T(r, G) \leq N_{2}(r, 0 ; F)+N_{2}(r, 0 ; G)+\bar{N}(r, \infty ; F)+\bar{N}(r, \infty ; G)+\bar{N}_{*}(r, \infty ; F, G)-m(r, 1 ; F)-$ $\bar{N}_{E}^{(3}(r, 1 ; G)-\bar{N}_{L}(r, 1 ; F)+S(r, F)+S(r, G)$.

Lemma 5. [15] Suppose that $F$ and $G$ be two nonconstant meromorphic functions and

$$
V=\left(\frac{F^{\prime}}{F-1}-\frac{F^{\prime}}{F}\right)-\left(\frac{G^{\prime}}{G-1}-\frac{G^{\prime}}{G}\right) .
$$

If $F, G$ share $(\infty, 0)$ and if $V=0$, then $F=G$.

Lemma 6. Let $f$ and $g$ be two nonconstant meromorphic functions, and let $n(\geq 1), k(\geq 1)$ and $m(\geq 0)$ be three integers. Suppose that $V$ is given as in $(2.3)$, where $F=\frac{\left(f^{n} P(f)\right)^{(k)}}{p(z)}, G=\frac{\left(g^{n} P(g)\right)^{(k)}}{p(z)}$, $P(w), p(z)$ are defined as in Theorem 1. If $V \not \equiv 0, F$ and $G$ share $(1,2), f$ and $g$ share $\infty$ IM, then the poles of $F$ and $G$ are zeros of $V$ and

$$
\begin{aligned}
(n+m+k-1) \bar{N}(r, \infty ; f \mid \geq 1)= & (n+m+k-1) \bar{N}(r, \infty ; g \mid \geq 1) \\
& \leq \bar{N}(r, 0 ; F)+\bar{N}(r, 0 ; G)+\bar{N}_{*}(r, 1 ; F, G) \\
& +S(r, f)+S(r, g) .
\end{aligned}
$$

Proof. We note that the order of the possible poles of $F$ and $G$ are at least $n+m+k$ as $f$ and $g$ share $(\infty, 0)$. Thus $F$ and $G$ share $(\infty, n+m+k-1)$. Now using Milloux theorem (see [6], p. 55) and Lemma 1, we obtain from the definition of $V$ that

$$
m(r, V)=S(r, f)+S(r, g) .
$$

Therefore

$$
\begin{aligned}
(n+m+k-1) \bar{N}(r, \infty ; f \mid \geq 1)= & (n+m+k-1) \bar{N}(r, \infty ; g \mid \geq 1) \\
= & (n+m+k-1) \bar{N}(r, \infty ; F \mid \geq n+m+k) \\
\leq & N(r, 0 ; V) \\
\leq & T(r, V)+O(1) \\
\leq & N(r, \infty ; V)+m(r, V)+O(1) \\
\leq & N(r, \infty ; V)+S(r, f)+S(r, g) \\
\leq & \bar{N}(r, 0 ; F)+\bar{N}(r, 0 ; G)+\bar{N}_{*}(r, 1 ; F, G) \\
& +S(r, f)+S(r, g) .
\end{aligned}
$$

This proves the lemma. 
Lemma 7. Let $f$ and $g$ be two transcendental meromorphic functions, and let $n(\geq 1), k(\geq 1)$, $m(\geq 0)$ be three integers. Suppose that $F$ and $G$ are defined as in Lemma 6 . If there exist two nonzero constants $c_{1}$ and $c_{2}$ such that $\bar{N}\left(r, c_{1} ; F\right)=\bar{N}(r, 0 ; G)$ and $\bar{N}\left(r, c_{2} ; G\right)=\bar{N}(r, 0 ; F)$, then $n \leq 3 k+m+3$.

Proof. By the second fundamental theorem of Nevanlinna we have

$$
\begin{aligned}
T(r, F) & \leq \bar{N}(r, 0 ; F)+\bar{N}\left(r, c_{1} ; F\right)+\bar{N}(r, \infty ; F)+S(r, F) \\
& \leq \bar{N}(r, 0 ; F)+\bar{N}(r, 0 ; G)+\bar{N}(r, \infty ; F)+S(r, F) .
\end{aligned}
$$

Using (2.1), (2.2), (2.4) and Lemma 1 we obtain

$$
\begin{aligned}
(n+m) T(r, f) \leq & T(r, F)-\bar{N}(r, 0 ; F)+N_{k+1}\left(r, 0 ; f^{n} P(f)\right) \\
& +O\{\log r\}+S(r, f) \\
\leq & \bar{N}(r, 0 ; G)+N_{k+1}\left(r, 0 ; f^{n} P(f)\right)+\bar{N}(r, \infty ; f) \\
& +O\{\log r\}+S(r, f) \\
\leq \quad & N_{k+1}\left(r, 0 ; f^{n} P(f)\right)+N_{k+1}\left(r, 0 ; g^{n} P(g)\right)+\bar{N}(r, \infty ; f) \\
& +k \bar{N}(r, \infty ; g)+O\{\log r\}+S(r, f)+S(r, g) \\
\leq \quad & (k+m+2) T(r, f)+(2 k+m+1) T(r, g) \\
& +O\{\log r\}+S(r, f)+S(r, g) .
\end{aligned}
$$

Similarly

$$
\begin{aligned}
(n+m) T(r, g) \leq \quad & (k+m+2) T(r, g)+(2 k+m+1) T(r, f) \\
& +O\{\log r\}+S(r, f)+S(r, g) .
\end{aligned}
$$

Combining (2.5), (2.6) and noting that $\log r=o(T(r, f))=o(T(r, g))$ we get

$$
(n-3 k-m-3)\{T(r, f)+T(r, g)\} \leq S(r, f)+S(r, g),
$$

which gives $n \leq 3 k+m+3$. This completes the proof of the lemma.

Q.E.D.

The following lemma can be proved in the line of the proof of Lemma 9 [18].

Lemma 8. Let $f$ and $g$ be two transcendental meromorphic functions, $p(z)$ be a nonconstant polynomial of degree $l$, and let $n(\geq 1), k(\geq 1)$ and $m(\geq 0)$ be three integers with $n>k+2 l$. If

$$
\left(f^{n} P(f)\right)^{(k)}\left(g^{n} P(g)\right)^{(k)}=p^{2}(z),
$$

where $P(w)$ is defined as in Theorem $\mathrm{G}$, then $P(w)$ is reduced to a nonzero monomial, namely $P(w)=a_{i} w^{i} \not \equiv 0$ for some $i \in\{0,1, \ldots, m\}$.

Lemma 9. Let $f$ and $g$ be two nonconstant meromorphic functions, $p(z)$ be a nonconstant polynomial of degree $l$, and let $n, m$ and $k$ be three positive integers with $n>k+2 l$. Futher assume that either $k, l$ are coprime or $k>l$ when $l \geq 2$. If

$$
\left(f^{n} P(f)\right)^{(k)}\left(g^{n} P(g)\right)^{(k)}=p^{2}(z),
$$

where $P(z)=a_{m} z^{m}$ or $P(z)=c_{0}$, then $f(z)=b_{1} e^{b Q(z)}, g(z)=b_{2} e^{-b Q(z)}$, where $b_{1}, b_{2}$ and $b$ are three constants satisfying $a_{m}^{2}((n+m) b)^{2}\left(b_{1} b_{2}\right)^{n+m}=-1$ or $c_{0}^{2}(n b)^{2}\left(b_{1} b_{2}\right)^{n}=-1$ and $Q(z)$ is same as in Theorem 1. 
Proof. Let $P(z)=a_{m} z^{m}$. The case $P(z)=c_{0}$ can be proved similarly. First we assume that $k=1$. Then (2.7) becomes

$$
\left(a_{m} f^{n+m}\right)^{\prime}\left(a_{m} g^{n+m}\right)^{\prime}=p^{2}(z) .
$$

Noting that $n>k+2 l$, we deduce from (2.8) that $f$ and $g$ have no zeros. We put

$$
f=e^{\alpha}, \quad g=e^{\beta},
$$

where $\alpha$ and $\beta$ are two nonconstant entire functions. Therefore from (2.8) we get

$$
a_{m}^{2}(n+m)^{2} \alpha^{\prime} \beta^{\prime} e^{(n+m)(\alpha+\beta)}=p^{2}(z) .
$$

From this it follows that $\alpha, \beta$ must be polynomials and $\alpha+\beta \equiv k_{1}$, where $k_{1}$ is a constant. Thus $\operatorname{deg}(\alpha)=\operatorname{deg}(\beta)$. Therefore $\alpha^{\prime}+\beta^{\prime} \equiv 0$ and

$$
a_{m}^{2}(n+m)^{2} \alpha^{\prime} \beta^{\prime} e^{(n+m) k_{1}}=p^{2}(z) .
$$

Simplifying we obtain $\alpha^{\prime}=b p(z)$ and $\beta^{\prime}=-b p(z)$, where $b(\neq 0)$ is a constant. This gives $\alpha=$ $b Q(z)+d_{1}$ and $\beta=-b Q(z)+d_{2}$, where $Q(z)$ is a polynomial without constant such that $Q^{\prime}(z)=p(z)$ and $d_{1}, d_{2}$ are constants. Therefore

$$
f=b_{1} e^{b Q(z)}, \quad g=b_{2} e^{-b Q(z)},
$$

where $b_{1}, b_{2}$ and $b$ are three constants satisfying

$$
a_{m}^{2}((n+m) b)^{2}\left(b_{1} b_{2}\right)^{n+m}=-1 .
$$

If $k \geq 2$ then $(2.7)$ becomes

$$
\left(a_{m} f^{n+m}\right)^{(k)}\left(a_{m} g^{n+m}\right)^{(k)}=p^{2}(z) .
$$

Since $f$ and $g$ are transcendental meromorphic function, from (2.10) we obtain

$$
N\left(r, 0 ;\left(a_{m} f^{n+m}\right)^{(k)}\right)=O\{\log r\} .
$$

From this and (2.9) we get

$$
N\left(r, \infty ; a_{m} f^{n+m}\right)+N\left(r, 0 ; a_{m} f^{n+m}\right)+N\left(r, 0 ;\left(a_{m} f^{n+m}\right)^{(k)}\right)=O\{\log r\} .
$$

Suppose that $\alpha$ is a transcendental entire function. Then by Lemma 3 we deduce that $\alpha$ is a polynomial, a contradiction. Next we assume that $\alpha, \beta$ are polynomials of degree $p_{1}$ and $p_{2}$ respectively. If $p_{1}=p_{2}=1$, then

$$
f=e^{A z+B}, \quad g=e^{C z+D},
$$

where $A(\neq 0), B, C(\neq 0)$ and $D$ are constants. So from (2.10) we obtain

$$
a_{m}^{2}(A C)^{k}(n+m)^{2 k} e^{(n+m)\{(A+C) z+(B+D)\}}=p^{2}(z),
$$

which is impossible. Thus $\max \left\{p_{1}, p_{2}\right\}>1$. We assume that $p_{1}>1$. Then $\left(a_{m} f^{n+m}\right)^{(k)}=$ $Q_{1} e^{(n+m) \alpha}$ and $\left(a_{m} g^{n+m}\right)^{(k)}=Q_{2} e^{(n+m) \beta}$, where $Q_{1}, Q_{2}$ are polynomials of degree $k\left(p_{1}-1\right)$ and $k\left(p_{2}-1\right)$ respectively. Therefore from (2.10) we obtain $\alpha+\beta \equiv k_{2}$, a constant, and therefore $p_{1}=p_{2}$ and $k\left(p_{1}-1\right)=l$. This shows that $l \geq k \geq 2$, contradicting with the assumption that $k, l$ are prime to each other. This proves the lemma.

Q.E.D. 


\section{Proof of the theorem}

Proof of Theorem 1. We discuss the following three cases separately.

Case (i) Let $P(z)=a_{m} z^{m}+a_{m-1} z^{m-1}+\ldots+a_{1} z+a_{0}$ is not a monomial. Suppose that $F$ and $G$ are defined as in Lemma 6 . Then $F, G$ are transcendental meromorphic functions that share $(1,2)$ and $(\infty, 0)$. Therefore

$$
\bar{N}_{*}(r, \infty ; F, G) \leq \bar{N}(r, \infty ; F \mid \geq n+m+k)=\bar{N}(r, \infty ; f \mid \geq 1) .
$$

If possible, we assume that $H \not \equiv 0$. Then $F \not \equiv G$. So from Lemma 5 we have $V \not \equiv 0$. From Lemma 1 and (2.1) we obtain

$$
\begin{aligned}
N_{2}(r, 0 ; F) \leq & N_{2}\left(r, 0 ;\left(f^{n} P(f)\right)^{(k)}\right)+S(r, f) \\
\leq & T\left(r,\left(f^{n} P(f)\right)^{(k)}\right)-(n+m) T(r, f)+N_{k+2}\left(r, 0 ; f^{n} P(f)\right) \\
& +S(r, f) \\
\leq & T(r, F)-(n+m) T(r, f)+N_{k+2}\left(r, 0 ; f^{n} P(f)\right) \\
& +O\{\log r\}+S(r, f) .
\end{aligned}
$$

Similarly

$$
\begin{aligned}
N_{2}(r, 0 ; G) \leq \quad & T(r, G)-(n+m) T(r, g)+N_{k+2}\left(r, 0 ; g^{n} P(g)\right) \\
& +O\{\log r\}+S(r, g) .
\end{aligned}
$$

Again by (2.2) we have

$$
N_{2}(r, 0 ; F) \leq N_{k+2}\left(r, 0 ; f^{n} P(f)\right)+k \bar{N}(r, \infty ; f)+S(r, f)
$$

and

$$
N_{2}(r, 0 ; G) \leq N_{k+2}\left(r, 0 ; g^{n} P(g)\right)+k \bar{N}(r, \infty ; g)+S(r, g) .
$$

From (3.1) and (3.2) we get

$$
\begin{aligned}
(n+m)\{T(r, f)+T(r, g)\} \leq \quad & T(r, F)+T(r, G)+N_{k+2}\left(r, 0 ; f^{n} P(f)\right) \\
& +N_{k+2}\left(r, 0 ; g^{n} P(g)\right)-N_{2}(r, 0 ; F)-N_{2}(r, 0 ; G) \\
& +O\{\log r\}+S(r, f)+S(r, g) .
\end{aligned}
$$


Then using Lemma 1, Lemma 4, (3.3) and (3.4) we obtain from (3.5)

$$
\begin{aligned}
(n+m)\{T(r, f)+T(r, g)\} \leq & N_{2}(r, 0 ; F)+N_{2}(r, 0 ; G)+2 \bar{N}(r, \infty ; F) \\
& +2 \bar{N}(r, \infty ; G)+2 \bar{N}_{*}(r, \infty ; F, G) \\
& +N_{k+2}\left(r, 0 ; f^{n} P(f)\right)+N_{k+2}\left(r, 0 ; g^{n} P(g)\right) \\
& -\bar{N}_{L}(r, 1 ; F)-\bar{N}_{L}(r, 1 ; G)+O\{\log r\} \\
& +S(r, f)+S(r, g) \\
\leq & 2 N_{k+2}\left(r, 0 ; f^{n} P(f)\right)+2 N_{k+2}\left(r, 0 ; g^{n} P(g)\right) \\
& +(k+2) \bar{N}(r, \infty ; f)+(k+2) \bar{N}(r, \infty ; g) \\
& +2 \bar{N}_{*}(r, \infty ; F, G)-\bar{N}_{*}(r, 1 ; F, G) \\
& +O\{\log r\}+S(r, f)+S(r, g) \\
\leq & 2(k+m+2)\{T(r, f)+T(r, g)\} \\
& +(k+2)(\bar{N}(r, \infty ; f)+\bar{N}(r, \infty ; g)) \\
& +2 \bar{N}_{*}(r, \infty ; F, G)-\bar{N}_{*}(r, 1 ; F, G) \\
& +O\{\log r\}+S(r, f)+S(r, g) .
\end{aligned}
$$

Using Lemma 2, Lemma 6 and noting that $f$ and $g$ are transcendental meromorphic functions we obtain from above

$$
\begin{aligned}
(n-3 & k-m-6)\{T(r, f)+T(r, g)\} \\
\leq & \frac{2}{n+m+k-1}\left[\bar{N}(r, 0 ; F)+\bar{N}(r, 0 ; G)+\bar{N}_{*}(r, 1 ; F, G)\right] \\
& -\bar{N}_{*}(r, 1 ; F, G)+S(r, f)+S(r, g) \\
\leq & \frac{2}{n+m+k-1}\left[N_{k+1}\left(r, 0 ; f^{n} P(f)\right)+k \bar{N}(r, \infty ; f)\right. \\
& \left.+N_{k+1}\left(r, 0 ; g^{n} P(g)\right)+k \bar{N}(r, \infty ; g)+\bar{N}_{*}(r, 1 ; F, G)\right] \\
& -\bar{N}_{*}(r, 1 ; F, G)+S(r, f)+S(r, g) .
\end{aligned}
$$

From this we obtain

$$
\begin{array}{r}
{[(n-3 k-m-6)(n+m+k-1)-(4 k+2 m+2)]\{T(r, f)+T(r, g)\}} \\
\leq S(r, f)+S(r, g),
\end{array}
$$

a contradiction as $n>\max \{3 k+m+6, k+2 l\}$.

Next we assume that $H=0$. Then

$$
\left(\frac{F^{\prime \prime}}{F^{\prime}}-\frac{2 F^{\prime}}{F-1}\right)-\left(\frac{G^{\prime \prime}}{G^{\prime}}-\frac{2 G^{\prime}}{G-1}\right)=0 .
$$

Integrating both sides of the above equality twice we get

$$
\frac{1}{F-1}=\frac{A}{G-1}+B
$$


where $A(\neq 0), B$ are constants. Now we consider the following three subcases.

Subcase (i) Let $B \neq 0$ and $A=B$. Then from (3.6) we get

$$
\frac{1}{F-1}=\frac{B G}{G-1} \text {. }
$$

If $B=-1$, then from (3.7) we obtain

$$
F G=1
$$

i.e.,

$$
\left(f^{n} P(f)\right)^{(k)}\left(g^{n} P(g)\right)^{(k)}=p^{2}(z)
$$

a contradiction by Lemma 8 .

If $B \neq-1$, from (3.7), we have $\frac{1}{F}=\frac{B G}{(1+B) G-1}$ and so $\bar{N}\left(r, \frac{1}{1+B} ; G\right)=\bar{N}(r, 0 ; F)$. Now from the second fundamental theorem of Nevanlinna, we get

$$
\begin{aligned}
T(r, G) & \leq \bar{N}(r, 0 ; G)+\bar{N}\left(r, \frac{1}{1+B} ; G\right)+\bar{N}(r, \infty ; G)+S(r, G) \\
& \leq \bar{N}(r, 0 ; F)+\bar{N}(r, 0 ; G)+\bar{N}(r, \infty ; G)+S(r, G)
\end{aligned}
$$

Using (2.1) and (2.2) we obtain from above inequality

$$
\begin{aligned}
T(r, G) \leq & N_{k+1}\left(r, 0 ; f^{n} P(f)\right)+k \bar{N}(r, \infty ; f)+T(r, G)+N_{k+1}\left(r, 0 ; g^{n} P(g)\right) \\
& -(n+m) T(r, g)+\bar{N}(r, \infty ; g)+O\{\log r\}+S(r, g) .
\end{aligned}
$$

Hence

$$
(n+m) T(r, g) \leq(2 k+m+1) T(r, f)+(k+m+2) T(r, g)+S(r, g) .
$$

In a similar manner we can get

$$
(n+m) T(r, f) \leq(k+m+2) T(r, f)+(2 k+m+1) T(r, g)+S(r, g) .
$$

Combining the above two inequality we obtain

$$
(n-3 k-m-3)\{T(r, f)+T(r, g)\} \leq S(r, f)+S(r, g),
$$

a contradiction as $n>\max \{3 k+m+6, k+2 l\}$.

Subcase (ii) Let $B \neq 0$ and $A \neq B$. Then from (3.6) we get $F=\frac{(B+1) G-(B-A+1)}{B G+(A-B)}$ and so $\bar{N}\left(r, \frac{B-A+1}{B+1} ; G\right)=\bar{N}(r, 0 ; F)$. Proceeding as in Subcase (i) we arrive at a contradiction.

Subcase (iii) Let $B=0$ and $A \neq 0$. Then from (3.6) $F=\frac{G+A-1}{A}$ and $G=A F-(A-1)$. If $A \neq 1$, we have $\bar{N}\left(r, \frac{A-1}{A} ; F\right)=\bar{N}(r, 0 ; G)$ and $\bar{N}(r, 1-A ; G)=\bar{N}(r, 0 ; F)$. So by Lemma 7 we have $n \leq 3 k+m+3$, a contradiction. Thus $A=1$ and hence $F=G$. Then

$$
\left[f^{n} P(f)\right]^{(k)}=\left[g^{n} P(g)\right]^{(k)} .
$$


Integrating we get

$$
\left[f^{n} P(f)\right]^{(k-1)}=\left[g^{n} P(g)\right]^{(k-1)}+c_{k-1},
$$

where $c_{k-1}$ is a constant. If $c_{k-1} \neq 0$, using Lemma 7 we deduce that $n \leq 3 k+m$, a contradiction. Thus $c_{k-1}=0$. Repeating k-times, we obtain

$$
f^{n} P(f)=g^{n} P(g) .
$$

Then

$$
f^{n}\left(a_{m} f^{m}+\ldots+a_{1} f+a_{0}\right)=g^{n}\left(a_{m} g^{m}+\ldots+a_{1} g+a_{0}\right) .
$$

Let $h=\frac{f}{g}$. If $h$ is a constant, by putting $f=g h$ in (3.9) we get

$$
a_{m} g^{n+m}\left(h^{n+m}-1\right)+a_{m-1} g^{n+m-1}\left(h^{n+m-1}-1\right)+\ldots+a_{0} g^{n}\left(h^{n}-1\right)=0,
$$

which implies $h^{d}=1$, where $d=\operatorname{gcd}(n+m, \ldots, n+m-i, \ldots, n+1, n), a_{m-i} \neq 0$ for some $i \in$ $\{0,1, \ldots, m\}$. Thus $f=t g$ for a constant $t$ such that $t^{d}=1, d=\operatorname{gcd}(n+m, \ldots, n+m-i, \ldots, n+1, n)$, $a_{m-i} \neq 0$ for some $i \in\{0,1, \ldots, m\}$.

If $h$ is not a constant, then from (3.9) we see that $f$ and $g$ satisfy the algebraic equation $R(f, g)=0$, where $R(f, g)$ is given by $(1.1)$.

Case (ii) Now we assume that $P(z)=a_{m} z^{m}$, where $a_{m}(\neq 0)$ is a complex constant. Let $F=$ $\frac{\left(a_{m} f^{n+m}\right)^{(k)}}{p(z)}$ and $G=\frac{\left(a_{m} g^{n+m}\right)^{(k)}}{p(z)}$. Then $F$ and $G$ are transcendental meromorphic functions that share $(1,2)$ and $(\infty, 0)$. Proceeding in the like manner as Case (i) above we obtain either $F G=1$ or $F=G$.

If $F G=1$, then

$$
\left(a_{m} f^{n+m}\right)^{(k)}\left(a_{m} g^{n+m}\right)^{(k)}=p^{2}(z) .
$$

So by Lemma 9 we obtain $f(z)=b_{1} e^{b Q(z)}, g(z)=b_{2} e^{-b Q(z)}$, where $b_{1}, b_{2}$ and $b$ are three constants satisfying $a_{m}^{2}((n+m) b)^{2}\left(b_{1} b_{2}\right)^{n+m}=-1$ and $Q(z)$ is same as in Theorem 1 . If $F=G$, then using Lemma 7 and proceeding similarly as in Case (i) we obtain $f=t g$ for a constant $t$ such that $t^{n+m}=1$.

Case (iii) Let $P(z)=c_{0}$. Taking $F=\frac{\left(c_{0} f^{n}\right)^{(k)}}{p(z)}, G=\frac{\left(c_{0} g^{n}\right)^{(k)}}{p(z)}$ and arguing similarly as in Case (ii) we obtain either $f(z)=b_{1} e^{b Q(z)}, g(z)=b_{2} e^{-b Q(z)}$, where $b_{1}, b_{2}$ and $b$ are three constants satisfying $c_{0}^{2}(n b)^{2}\left(b_{1} b_{2}\right)^{n}=-1, Q(z)$ is same as in Theorem 1 or $f=t g$ for a constant $t$ satisfying $t^{n}=1$. This completes the proof of the theorem.

Q.E.D.

\section{References}

[1] T. C. Alzahary and H. X. Yi, Weighted value sharing and a question of I. Lahiri, Complex Var. Theory Appl., 49(2004), 1063-1078.

[2] A. Banerjee, Uniqueness of meromorphic functions sharing two sets with finite weight, Portugal. Math. (N.S.) 65(2008), 81-93. 
[3] W. Bergweiler and A. Eremenko, On the singularities of the inverse to a meromorphic function of finite order, Rev. Mat. Iberoam, 11(1995), 355-373.

[4] H. H. Chen and M. L. Fang, On the value distribution of $f^{n} f^{\prime}$, Sci. China Ser. A 38(1995), 789-798.

[5] M. L. Fang and H. L. Qiu, Meromorphic functions that share fixed points, J. Math. Anal. Appl., 268(2002), 426-439.

[6] W. K. Hayman, Meromorphic functions, The Clarendon Press, Oxford (1964).

[7] I. Lahiri, Weighted value sharing and uniqueness of meromorphic functions, Complex Var. Theory Appl., 46(2001), 241-253.

[8] X. M. Li and H. X. Yi, Uniqueness of meromorphic functions whose certain nonlinear differential polynomials share a polynomial, Comput. Math. Appl., 62(2011), 539-550.

[9] P. Sahoo, Meromorphic functions that share fixed points with finite weights, Bull. Math. Anal. Appl., 2(2010), 106-118.

[10] P. Sahoo, Uniqueness and weighted sharing of meromorphic functions, Ann. Polon. Math., 100(2011), 127-145.

[11] J. Xia and Y. Xu, Uniqueness and differential polynomials of meromorphic functions sharing one value, Filomat J. Math., 25(2011), 185-194.

[12] J. F. Xu, F. Lu and H.X. Yi, Fixed points and uniqueness of meromorphic functions, Comput. Math. Appl., 59(2010), 9-17.

[13] C. C. Yang, On deficiencies of differential polynomials II, Math. Z., 125(1972), 107-112.

[14] C. C. Yang and X. H. Hua, Uniqueness and value sharing of meromorphic functions, Ann. Acad. Sci. Fenn. Math. 22(1997), 395-406.

[15] H. X. Yi, Meromorphic functions that share three sets, Kodai Math. J., 20(1997), 22-32.

[16] H. X. Yi and C. C. Yang, Uniqueness theory of meromorphic functions, Science Press, Beijing, 1995.

[17] J. L. Zhang and L. Z. Yang, Some results related to a conjecture of R. Bruck, J. Inequal. Pure Appl. Math., 8(2007), Art. 18.

[18] X. B. Zhang and J. F. Xu, Uniqueness of meromorphic functions sharing a small function and its applications, Comput. Math. Appl., 61(2011), 722-730. 